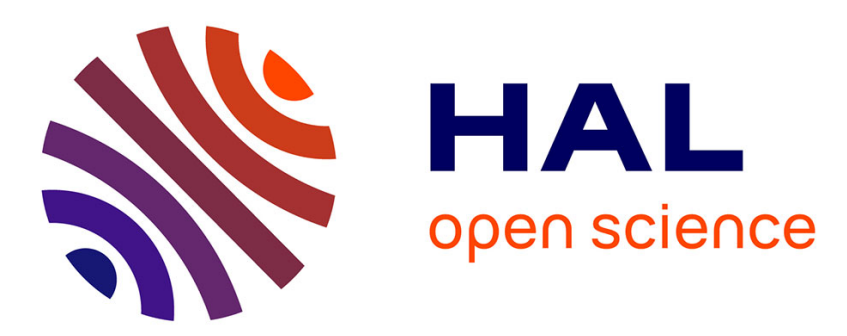

\title{
Heavy-Ion Fusion Mechanism And Predictions of Super-Heavy Elements Production
}

\author{
Y. Abe, Caiwan Shen, D. Boilley, B.G. Giraud, G. Kosenko
}

\section{To cite this version:}

Y. Abe, Caiwan Shen, D. Boilley, B.G. Giraud, G. Kosenko. Heavy-Ion Fusion Mechanism And Predictions of Super-Heavy Elements Production. Nuclear Structure and Dynamics (NSD09), May 2009, Dubrovnik, Croatia. pp.128-131, 10.1063/1.3232052 . in2p3-00384520

HAL Id: in2p3-00384520

https://hal.in2p3.fr/in2p3-00384520

Submitted on 2 Sep 2009

HAL is a multi-disciplinary open access archive for the deposit and dissemination of scientific research documents, whether they are published or not. The documents may come from teaching and research institutions in France or abroad, or from public or private research centers.
L'archive ouverte pluridisciplinaire HAL, est destinée au dépôt et à la diffusion de documents scientifiques de niveau recherche, publiés ou non, émanant des établissements d'enseignement et de recherche français ou étrangers, des laboratoires publics ou privés. 


\title{
Heavy-Ion Fusion Mechanism And Predictions Of Super-Heavy Elements Production
}

\author{
Yasuhisa Abe $^{\mathrm{a}}$, Caiwan Shen ${ }^{\mathrm{b}}$, David Boilley ${ }^{\mathrm{c}}$, Bertrand G. Giraud ${ }^{\mathrm{d}}$ \\ and Grigory Kosenko ${ }^{\mathrm{e}}$ \\ ${ }^{a} R C N P$, Osaka University, Ibaraki (Osaka), 567-0047, Japan. \\ ${ }^{b}$ School of Science, Huzhou Teachers College, Huzhou (Zhejiang), 313000, China, \\ ${ }^{\circ}$ GANIL,CEA/DSM-CNRS/IN2P3, BP 55027, F-14076, France, \\ and Univ. Caen, BP 5186, F-14032 Caen, France, \\ ${ }^{d} I P T, C E A / D S M, C E A-S a c l a y$, Gif-sur-Yvette, F-91191, France, \\ ${ }^{e}$ Department of Physics, Omsk University, Omsk, RU-644077, Russia
}

\begin{abstract}
Fusion process is shown to firstly form largely deformed mono-nucleus and then to undergo diffusion in two-dimensions with the radial and mass-asymmetry degrees of freedom. Examples of prediction of residue cross sections are given for the elements with $Z=117$ and 118 .
\end{abstract}

Keywords: Heavy-ion Fusion; Fusion hindrance; Super-heavy elements; Cross section.

PACS: 25.70, Jj, 25.70. Lm, 27.90. + b

\section{INTRODUCTION}

Theoretical prediction of optimum incident system, optimum incident energy, and absolute value of maximum cross section for Super-Heavy Elements (SHE) production is a long-standing challenging problem in nuclear physics. It becomes more and more important when we go to heavier elements, because residue cross section becomes smaller and smaller, down to the order of $\mathrm{pb}$ to $\mathrm{fb}$. Why such extremely small cross sections? One immediately thinks of fragility of SHE, that is, the fact that there is no barrier against fission within the Liquid Drop Model (LDM), and only so-called shell correction energy in the ground state sustains nucleus of SHE against fission decay, as is understood by the fissility being close to 1 . The feature is correctly taken into account through Ignatyuk prescription of the level density parameter. $^{1,2}$ As expected, the survival probability for SHE is very small, even if compound nucleus is formed. The small cross section, however, is not only due to the survival probability, but also due to small fusion probability, which is expected from so-called fusion hindrance. ${ }^{3,4}$ Existence of the hindrance has been known experimentally since many years ago, but its mechanism was not understood yet.

Recently the mechanism has been clarified. ${ }^{5,6,7}$ The theory is based on the observation that di-nucleus configuration formed by the incident projectile and target is located outside the fission saddle or the ridge-line, because the configuration has a very large deformation as a compound nucleus, while the saddle point configuration is 
close to the spherical shape in heavy nuclei with fissility close to 1 . One more essential point in the theory is an assumption that dissipation is very strong, strong enough for the relative kinetic energy to dissipate already at the contact distance of the projectile and the target, which is confirmed with the Surface Friction Model (SFM). ${ }^{8,9}$ Thus, we employ Smoluchowski or over-damped Langevin equation for the multidimensional fusion dynamics for overcoming of the saddle point or the ridge-line to the spherical compound nucleus.

\section{DYNAMICS FROM DI-NUCLEUS TO MONO-NUCLEUS ${ }^{10,11}$}

For the description of nuclear shapes of the composite system formed by the incident channel, we employ Two-Center Parameterization (TCP), which encompasses dinucleus as well as mono-nucleus configurations. ${ }^{12,13,14}$

There are three essential parameters: distance between two centers of the oscillator potentials $\mathrm{R}$, mass-asymmetry $\alpha$, and neck correction $\varepsilon$. The neck correction $\varepsilon$ is defined as a ratio between the smoothed peak height at the connection point of the right and left oscillator potentials and the height of the potential without correction, i.e., that of the potential spike at the connection point. So, $\varepsilon$ can vary between 1 and 0 . The value 1 corresponds to the smoothed peak with the same height as the original spike, while the value 0 does to no peak, i.e., to a single wide flat potential. In other words, the former describes the touching configuration of the incident channel, while the latter does mono-nucleus with very large deformation. In the TCP, the three degrees of freedom are almost independent, especially in di-nucleus configurations, though they are not normal modes. Thus, it is meaningful to analyze each degree of freedom separately. Of course, there are couplings between them, and friction tensor also induces couplings, for which we use so-called One-Body Dissipation model (OBD). ${ }^{15}$ The coupling effects will be discussed elsewhere, starting with multidimensional Smoluchowski equation. ${ }^{16}$

Firstly, we take up the case with mass-symmetric entrance channel, which makes the problem simpler with only two degrees of freedom left. The radial motion is already solved and analyzed in detail. ${ }^{17,18}$ Fusion probability, i.e., a probability for passing over the saddle point into the spherical shape is shown to be given by the fluctuation of Langevin trajectories, or by a tail of diffusion, and to be well approximated by an error function. In cases with strong dissipation such as OBD, the function can be approximated by an Arrhenius function, $\exp \left(-V_{\text {sad }} / T\right)$, where $V_{\text {sad }}$ denotes the saddle point height measured from the energy of the touching configuration and $T$ the temperature of the composite system. This clearly explains the feature of the fusion hindrance, i.e., an extremely small and slow increase of the fusion probability known experimentally. ${ }^{3,4}$ Here, it is worth to notice that $V_{\text {sad }}=0.0$ defines a border between the normal and the hindered fusions. Interestingly, the border line obtained is found to be consistent with the measured data on ${ }^{100} \mathrm{Mo}+{ }^{100} \mathrm{Mo}$ (normal) and ${ }^{110} \mathrm{Pd}+{ }^{110} \mathrm{Pd}$ (hindered) systems. ${ }^{19}$ Next, the time evolution of the radial fusion was analyzed, which shows that the fusion process is undertaken in time scale around several in unit of $\hbar / \mathrm{MeV} .^{18}$

During the diffusion process in the radial motion, how does the neck degree of freedom evolve? In order to answer that question, we solve the neck motion with the 
starting point $\varepsilon$ equal to 1.0 . The radial variable is fixed at the contact distance of two nuclei for the moment. The potential for $\varepsilon$ is calculated with LDM, which is almost linear, and the friction is calculated with OBD. Corresponding 1-dimensional Smoluchowski equation was already solved by him. ${ }^{20}$

We apply it to our present case and find that the neck degree of freedom quickly reaches to the equilibrium distribution in the space $[0.0,1.0]$, in one order of magnitude shorter than the time scale of the radial diffusion. ${ }^{10}$ The average value of the neck variable is about 0.1 , near the bottom of the potential. This indicates that the fusion process proceeds to firstly filling-out of the neck cleft or crevice of the dinucleus toward the formation of the mono-nucleus with the very large deformation, and then to diffusion in the radial degree of freedom.

The mass-asymmetry degree of freedom is also analyzed in the same way for $48 \mathrm{Ca}$ induced reactions, and turns out that its time scale is close to that of the radial one, not to the neck one. ${ }^{11}$

\section{PRELIMINARY RESULTS OF RESIDUE CROSS SECTIONS}

In realistic calculations of fusion probability, we numerically solve two-dimensional Langevin equation for radial and mass-asymmetry degrees of freedom ${ }^{21}$ with neck parameter being fixed at the average value of 0.1 . We also need to know the probability for overcoming of the usual Coulomb barrier in the entrance channel. There are two ways to employ: the empirical formula for capture cross sections ${ }^{22}$ and Langevin calculations of trajectories with SFM. ${ }^{8,9}$ As for the survival probability, we use the theory of statistical decay for particle emission and fission decay. ${ }^{23,24}$
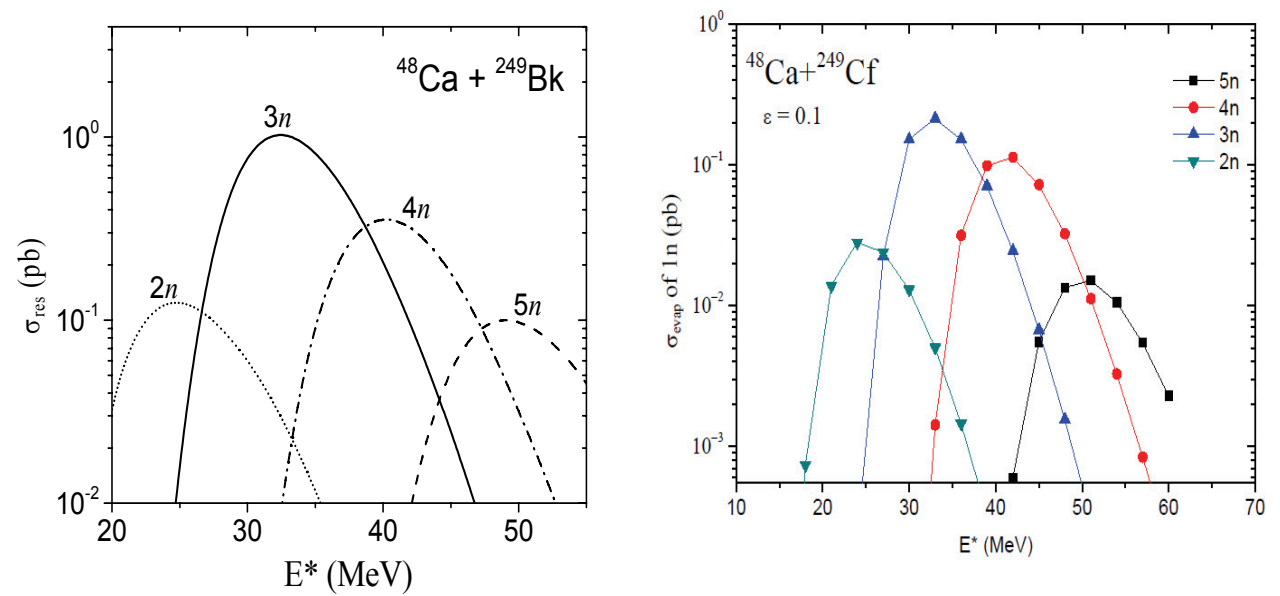

FIGURE 1. Predictions of $x n$ residue cross sections for $Z=117$ and 118 elements. No arbitrary parameter is introduced except the reduction factor for the shell correction energy in the calculations of the survival probabilities

Preliminary studies are made on ${ }^{48} \mathrm{Ca}+\mathrm{Bk}$ isotopes ${ }^{25}$ as well as ${ }^{48} \mathrm{Ca}+{ }^{249} \mathrm{Cf}$ systems, where mass table predicted by P. Möller et al. is used. ${ }^{26}$ In Fig. 1, the results 
for the systems for $Z=117$ and 118 are shown, where the empirical formula is used for the capture probability with certain modification of the parameters suitable for ${ }^{48} \mathrm{Ca}$ induced reactions, and a part of the code HIVAP for the statistical decay.

Since there are still ambiguities in fusion probabilities, we premise that absolute values of residues cannot be reproduced. We adjust it expediently by reducing the shell correction energy, i.e., fission barrier height and thus the survival probability. The calibration of the factor is made with the Dubna data on ${ }^{48} \mathrm{Ca}+{ }^{248} \mathrm{Cm}$ system. ${ }^{27}$ The factor turns out to be 0.45 , which we keep in use for the other systems. ${ }^{25}$ In order to eliminate the factor, more detailed investigations are necessary on fusion dynamics in very heavy systems, which are under way. ${ }^{16}$

\section{ACKNOWLEDGMENTS}

The present work is supported by JSPS grant No. 18540268 and National Science Foundation of China grant No. 10675046.

\section{REFERENCES}

1. A.V. Ignatyuk et al., Sov. J. Nucl. Phys. 21, 255 (1975).

2. Y. Abe and B. Bouriquet, Acta Physica Polonica B34, 1927-1945 (2003).

3. W. Westmeier et al., Phys. Lett. B117, 163-166 (1982).

4. W. Reisdorf, J. Phys. G; Nucl. Part. 20, 1297 (1994).

5. Y. Abe, Eur. Phys. J. A13, 143 (2002).

6. Y. Abe et al., Prog. Theor. Phys. Suppl. No. 146, 104- 109 (2002).

7. Y. Abe et al., Acta Physica Polonica B34, 2091-2105 (2003).

8. D.H.E. Gross and H. Kalinowski, Phys. Reports 45, 175 (1978).

9. G. Kosenko, C.W. Shen, and Y. Abe, J. Nucl. Radiochem. Sci. 3, 19-22 (2002).

10. Y. Abe et al., Intern. J. Mod. Phys. E17, 2214-2220 (2008).

11. Y. Abe et al., to appear in Intern. J. Mod. Phys.

12. J. Maruhn and W. Greiner, Z. Phys. 251, 431 (1972).

13. S. Suekane et al., JAERI-memo 5918 (1974).

14. A. Iwamoto et al., Prog. Theor. Phys. 55, 115 (1976).

15. J. Blocki et al., Ann. Phys. 113, 330 (1978).

16. D. Boilley et al., publication under preparation.

17 Y. Abe et al., Phys. Rev. E61, 1125-1133 (2000).

18. D. Boilley, Y. Abe and J.D. Bao, Eur. Phys. J. A18, 627-631 (2003).

19. C. Shen et al., to appear in Science in China Series $G$.

20 M. V. Smoluchowski, Physik Zeit. 17, 585 (1916).

21. C. Shen, G. Kosenko and Y. Abe, Phys. Rev. C66, 061602 (2002),

B. Bouriquet, G. Kosenko, and Y. Abe, Eur. Phys. J. A22, 9-12 (2004).

22. K. Siwek-Wilczynska, E. Siemaszko and J. Wilczynski, Acta Physica Polonica B33, 451 (2002).

23. HIVAP code, W. Reisdorf.

24. B. Bouriquet, D. Boilley and Y. Abe, Comp. Phys. Comm. 159, 1-18 (2004).[KEWPIE I]

A. Marchix, PhD thesis, Univ. Caen, 2007. [KEWPIE II]

25. C. Shen et al., Intern. J. Mod. Phys. E17, suppl. 66-79 (2008).

26. P. Möller et al., Atom. Data Nucl. Data Tables 59, 185 (1995).

27. Yu. Ts. Oganessian et al., Phys. Rev. C74 044602 (2006), and references therein. 